\title{
Determination of Karsch Coefficients for 2-colour QCD
}

\author{
Seamus Cotter* \\ Department of Mathematical Physics, National University of Ireland Maynooth, \\ Maynooth, County Kildare, Ireland \\ E-mail: seamus.cotter@nuim.ie

\section{Pietro Giudice} \\ Universität Münster, Institut für Theoretische Physik, Münster, Germany \\ E-mail: p.giudice@uni-muenster.de

\section{Simon Hands} \\ Department of Physics, College of Science, Swansea University, Swansea, United \\ Kingdom \\ E-mail: s.hands@swansea.ac.uk

\section{Jon-Ivar Skullerud} \\ Department of Mathematical Physics, National University of Ireland Maynooth, \\ Maynooth, County Kildare, Ireland \\ E-mail: jonivar@thphys.nuim.ie
}

We give an update of results from two-colour, two-flavour QCD. Using a Wilson fermion action we calculate thermodynamic quantities as a function of chemical potential $\mu$. Calculating the Karsch Coefficients non-perturbatively gives us access to the derivative method. Compared to our previously published results, we have improved our analysis leading to revised and more accurate estimates for the renormalised energy density, pressure and the trace anomaly.

31st International Symposium on Lattice Field Theory LATTICE 2013

July 29 August 3, 2013

Mainz, Germany

${ }^{*}$ Speaker. 


\section{Introduction}

As part of a larger study on two-colour, two-flavour QCD, we use the derivative method to calculate thermodynamic quantities, in particular the renormalised energy density. The major stumbling block is the accurate calculation of the Karsch coefficients [1]. These are defined as the derivative of input parameters with respect to measured observables. In this case the input parameters are the gauge coupling $\beta$, the hopping parameter $\kappa$ and the input gauge and quark anisotropies $\gamma_{g}$ and $\gamma_{q}$ of our action $S=S_{G}+S_{Q}+S_{J}$. This consists of a non-improved Wilson gauge and fermion action along with a diquark source action which serves to lift the low lying eigenmodes of the Dirac operator:

$$
\begin{aligned}
S_{G} & =-\frac{\beta}{N_{c}}\left[\frac{1}{\gamma_{g}} \sum_{x, i<j} \operatorname{Re} \operatorname{Tr} U_{i j}(x)+\gamma_{g} \sum_{x i} \operatorname{Re} \operatorname{Tr} U_{i 0}(x)\right] \\
S_{Q} & =\sum_{x, \alpha}\left[\bar{\psi}^{\alpha}(x) \psi^{\alpha}(x)+\gamma_{q} \kappa \bar{\psi}^{\alpha}(x)\left(D_{0} \psi\right)^{\alpha}(x)\right]+\kappa \sum_{x, \alpha, i} \bar{\psi}^{\alpha}(x)\left(D_{i} \psi\right)^{\alpha}(x), \\
S_{J} & =\kappa j \sum_{x}\left[\psi^{2 t r}(x) C \gamma_{5} \tau_{2} \psi^{1}(x)-\bar{\psi}^{1}(x) C \gamma_{5} \tau_{2} \bar{\psi}^{2 t r}(x)\right] .
\end{aligned}
$$

We define $\beta_{s}=\frac{\beta}{\gamma_{g}}, \beta_{t}=\gamma_{g} \beta, \kappa_{s}=\kappa$ and $\kappa_{t}=\gamma_{q} \kappa$. We then calculate the Karsch coefficients non-perturbatively by measuring the lattice spacing $a_{s}$, the pion/rho meson mass ratio $M=\frac{m_{\pi}}{m_{\rho}}$, and the measured gauge $\xi_{g}$ and quark $\xi_{q}$ anisotropies on several ensembles of anisotropic and isotropic lattices across a range of values for $\beta, \kappa, \gamma_{g}$ and $\gamma_{q}$ taken around the central set $\beta=1.9, \kappa=0.168$ with $\gamma_{g}=\gamma_{q}=1$, listed in Table 1. We define the average anisotropy $\xi^{+}=\frac{1}{2}\left(\xi_{g}+\xi_{q}\right)$ and the anisotropy mismatch $\xi^{-}=\frac{1}{2}\left(\xi_{g}-\xi_{q}\right)$, to ensure we are working along a line of constant physics. As all thermodynamic quantities are extrapolated to diquark source $j=0$, the diquark term in the action plays no further role.

Further details about the initial setup are given in an earlier paper [2]. In that paper we overlooked the quark number density term of the energy density which we include now. A consequence of the inclusion of this term is that the energy density can be seen to rely almost totally on this term as the fermionic and gluonic contributions are small and nearly cancel. As a result the energy density becomes almost Karsch coefficient independent. This behaviour was conjectured in an earlier paper [3]. We also calculate the pressure which can be compared to results that used the integral method in [2] and the trace anomaly.

To calculate the spatial lattice spacing $a_{s}$, we use the static quark potential, and to calculate the gauge anisotropy $\xi_{g}$ we use the sideways potential [4]. For the pion/rho meson mass ratio $M=\frac{m_{\pi}}{m_{\rho}}$ and the quark anisotropy $\xi_{q}$ we use the meson dispersion. The results are shown in Table 1 and Figures 1 and 2. Using these measurement results allows for a four dimensional fit of the measured values for $a_{s}, M, \xi^{+}$and $\xi^{-}$as a function of the input parameters $\beta, \kappa, \gamma_{q}$ and $\gamma_{g}$. Inverting the resulting $4 \times 4$ matrix gives us the Karsch coefficients shown in Table 2 and 3. 


\begin{tabular}{|cccccc|cccc|}
\hline$\beta_{s}$ & $\beta_{t}$ & $\kappa_{s}$ & $\kappa_{t}$ & $\gamma_{g}$ & $\gamma_{q}$ & $\xi_{g}$ & $\xi_{q}$ & $M=\frac{m_{\pi}}{m_{\rho}}$ & $a_{s}(\mathrm{fm})$ \\
\hline \hline 1.90 & 1.90 & 0.1680 & 0.1680 & 1.0 & 1.0 & $0.968_{-2}^{+2}$ & $1.035_{-10}^{+8}$ & $0.798_{-9}^{+4}$ & $0.178_{-6}^{+4}$ \\
\hline 2.37 & 1.52 & 0.1680 & 0.1680 & 0.8 & 1.0 & $0.721_{-2}^{+2}$ & $0.999_{-9}^{+8}$ & $0.807_{-3}^{+3}$ & $0.177_{-3}^{+4}$ \\
1.27 & 2.83 & 0.1680 & 0.1680 & 1.5 & 1.0 & $1.321_{-5}^{+6}$ & $1.278_{-3}^{+21}$ & $0.633_{-12}^{+9}$ & $0.125_{-5}^{+3}$ \\
1.90 & 1.90 & 0.1800 & 0.1570 & 1.0 & 0.87 & $0.747_{-4}^{+4}$ & $0.875_{-34}^{+24}$ & $0.711_{-14}^{+19}$ & $0.107_{-5}^{+2}$ \\
1.90 & 1.90 & 0.1470 & 0.1920 & 1.0 & 1.3 & $1.146_{-4}^{+4}$ & $1.513_{-12}^{+15}$ & $0.946_{-1}^{+1}$ & $0.229_{-12}^{+7}$ \\
\hline 1.80 & 1.80 & 0.1740 & 0.1740 & 1.0 & 1.0 & $0.989_{-3}^{+4}$ & $1.028_{-14}^{+16}$ & $0.770_{-6}^{+5}$ & $0.177_{-7}^{+5}$ \\
1.90 & 1.90 & 0.1685 & 0.1685 & 1.0 & 1.0 & $0.945_{-5}^{+5}$ & $1.020_{-11}^{+9}$ & $0.759_{-13}^{+11}$ & $0.153_{-18}^{+7}$ \\
2.00 & 2.00 & 0.1620 & 0.1620 & 1.0 & 1.0 & $0.921_{-5}^{+4}$ & $0.992_{-9}^{+10}$ & $0.819_{-6}^{+7}$ & $0.166_{-2}^{+1}$ \\
2.00 & 2.00 & 0.1630 & 0.1630 & 1.0 & 1.0 & $0.881_{-5}^{+5}$ & $1.008_{-6}^{+9}$ & $0.756_{-7}^{+13}$ & $0.148_{-1}^{+1}$ \\
\hline
\end{tabular}

Table 1: Ensemble parameters and measured values for the anisotropies, mass ratio and lattice spacing.

\section{Improvements to the determination}

Apart from minor alterations to fit ranges, one area where we immediately focused our attention was the meson dispersion. Two of the columns of the $4 \times 4$ matrix consist of results from the meson dispersion, any minor improvement could potentially give a large overall improvement. For the mass fits at zero momentum, this improvement came from a tightening of the fit range. For the meson dispersion after a similar analysis and study of the fit ranges an improvement was also seen. On top of this we also switched from using the continuum definition of the dispersion relation:

$$
a_{t}^{2} E^{2}(p)=a_{t}^{2} m_{\pi}^{2}+\frac{p^{2}}{a_{s}^{2} \xi_{q}^{2}}, \quad \text { where } p^{2}=p_{x}^{2}+p_{y}^{2}+p_{z}^{2}
$$

to the lattice version following [5]:

$$
p^{2}=\frac{4}{a^{2}}\left\{\sin ^{2}\left(\frac{p_{x} a}{2}\right)+\sin ^{2}\left(\frac{p_{y} a}{2}\right)+\sin ^{2}\left(\frac{p_{x} a}{2}\right)\right\}
$$

This takes into account the discrete values of the momentum on the lattice. These two improvement resulted in lower error bars across the board, which can be seen in the latest results for the Karsch coefficients below. The improved results for the Karsch coefficients (Table 3) show a reduction in the size of errors from the earlier determination (Table 2).

\begin{tabular}{|c|cccc|}
\hline$c_{i}$ & $\frac{\partial c_{i}}{\partial \xi_{+}}$ & $a \frac{\partial c_{i}}{\partial a}$ & $M \frac{\partial c_{i}}{\partial M}$ & $\frac{\partial c_{i}}{\partial \xi_{-}}$ \\
\hline \hline$\gamma_{g}$ & $0.90_{-0.14}^{+0.04}$ & $-0.51_{-0.10}^{+0.19}$ & $0.13_{-0.58}^{+0.32}$ & $1.4_{-1.6}^{+1.2}$ \\
$\gamma_{q}$ & $0.13_{-0.05}^{+0.40}$ & $0.22_{-0.70}^{+0.12}$ & $-0.55_{-0.29}^{+2.11}$ & $-2.9_{-0.6}^{+5.7}$ \\
$\beta$ & $0.59_{-1.37}^{+0.24}$ & $-1.4_{-0.5}^{+2.3}$ & $3.7_{-7.0}^{+1.9}$ & $8_{-19}^{+8}$ \\
$\kappa$ & $-0.05_{-0.02}^{+0.07}$ & $0.08_{-0.09}^{+0.02}$ & $-0.22_{-0.08}^{+0.35}$ & $-0.39_{-0.23}^{+0.88}$ \\
\hline
\end{tabular}

Table 2: Original Karsch coefficients determination. 


\begin{tabular}{|c|cccc|}
\hline$c_{i}$ & $\frac{\partial c_{i}}{\partial \xi_{+}}$ & $a \frac{\partial c_{i}}{\partial a}$ & $M \frac{\partial c_{i}}{\partial M}$ & $\frac{\partial c_{i}}{\partial \xi_{-}}$ \\
\hline \hline$\gamma_{g}$ & $0.79_{-0.08}^{+0.04}$ & $-0.48_{-0.14}^{+0.09}$ & $0.08_{-0.08}^{+0.23}$ & $1.04_{-0.15}^{+0.46}$ \\
$\gamma_{q}$ & $0.39_{-0.03}^{+0.02}$ & $-0.03_{-0.04}^{+0.04}$ & $0.28_{-0.08}^{+0.09}$ & $-0.47_{-0.15}^{+0.23}$ \\
$\beta$ & $-0.27_{-0.19}^{+0.08}$ & $-0.86_{-0.36}^{+0.22}$ & $1.49_{-0.19}^{+0.75}$ & $1.94_{-0.29}^{+1.69}$ \\
$\kappa$ & $-0.01_{-0.01}^{+0.01}$ & $0.05_{-0.02}^{+0.02}$ & $-0.12_{-0.05}^{+0.14}$ & $-0.11_{-0.11}^{+0.02}$ \\
\hline
\end{tabular}

Table 3: Improved Karsch coefficients determination.
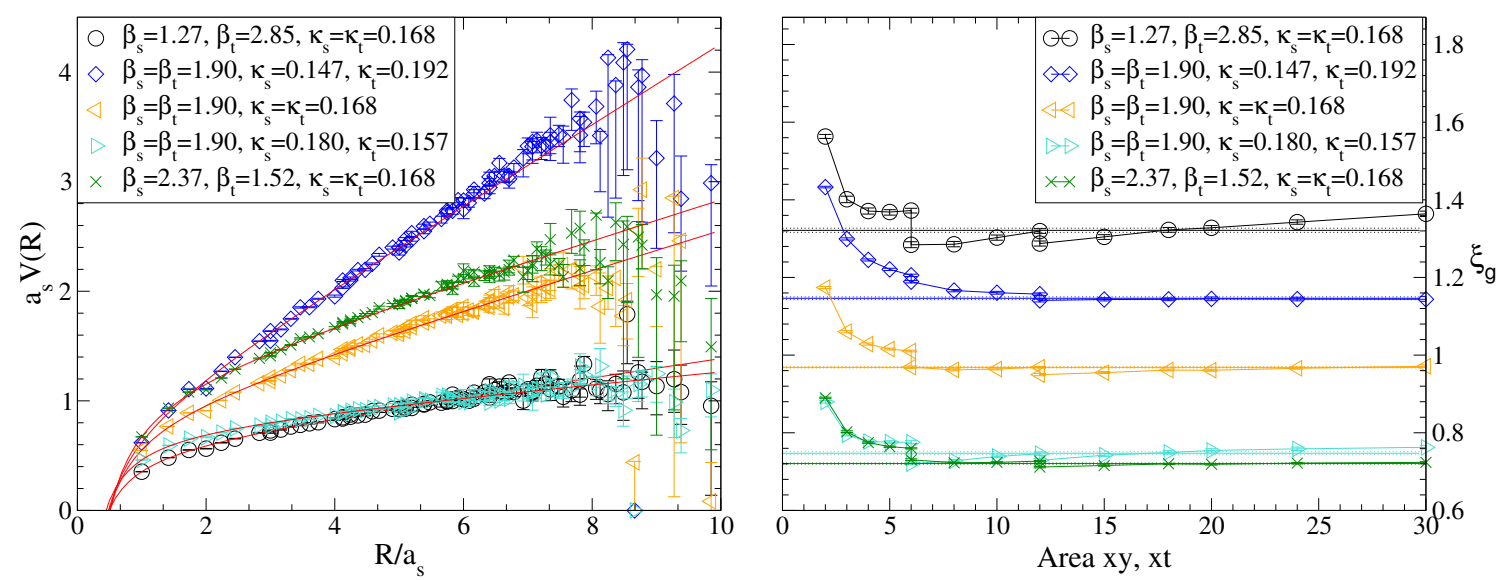

Figure 1: Static quark potential (left) and Sideways potential results (right) are shown for the central set and the anisotropic sets.
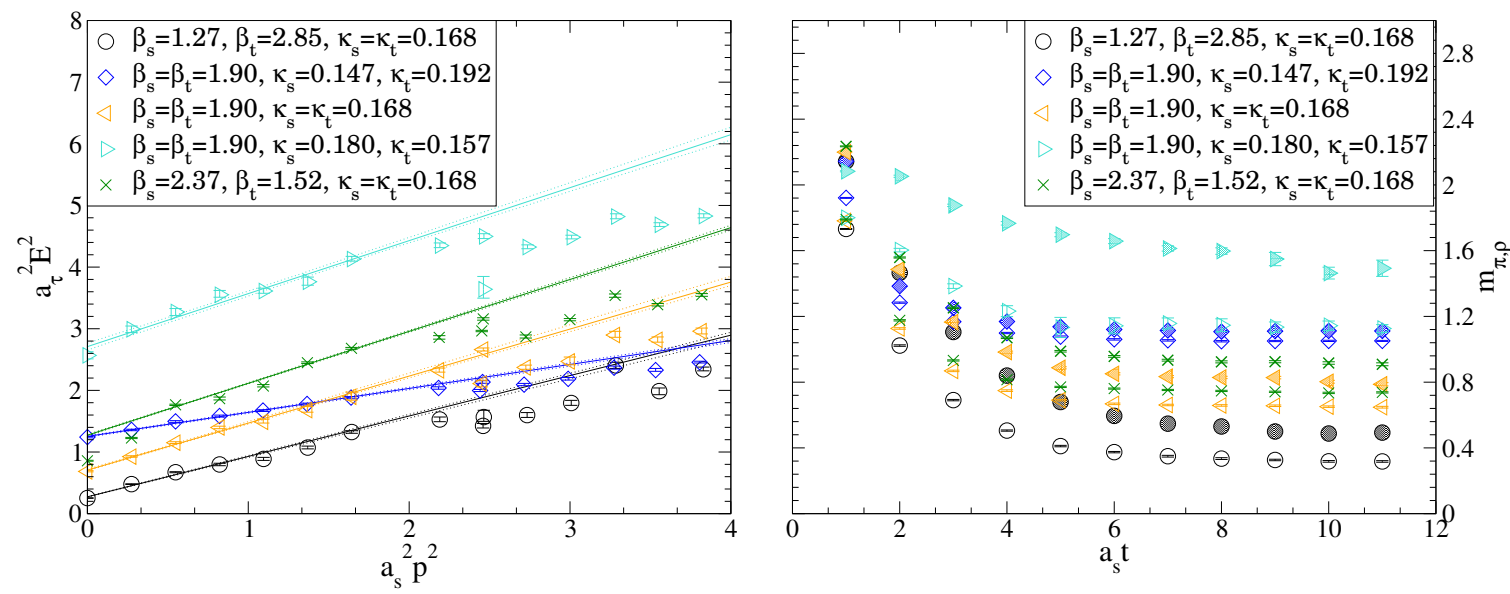

Figure 2: The pion dispersion (left) and the effective mass (right) of the pion (empty) and rho (shaded) mesons for the central set and the anisotropic sets. 


\section{Equation of State}

The partial derivatives must be taken with all other physical parameters fixed, which means the physical quark mass, and therefore the mass ratio $M=\frac{m_{\pi}}{m_{\rho}}$ are kept fixed. The energy density can be derived using the standard thermodynamic relations:

$$
\begin{gathered}
\Omega=E-T \mathbb{S}-\mu_{q} N_{q}=-p V=-T \ln \mathcal{Z} \\
p=\left.\frac{\partial(T \ln \mathcal{Z})}{\partial V}\right|_{T}, \quad \varepsilon=\left.\frac{E}{V}\right|_{V}, \mathbb{S}=\left.\frac{\partial(T \ln \mathcal{Z})}{\partial T}\right|_{V}, \quad n_{q}=\frac{N_{q}}{V}=\frac{1}{V} \frac{\partial(T \ln \mathcal{Z})}{\partial \mu},
\end{gathered}
$$

which gives us

$$
\begin{aligned}
\varepsilon & =\varepsilon_{g}+\varepsilon_{q}+\mu_{q} n_{q}=-\frac{T}{V}\left\langle\xi \frac{\partial S}{\partial \xi}\right\rangle+\mu_{q} n_{q}, \\
& =\frac{3}{N_{s}^{3} a_{s}^{3} N_{t} a_{t} N_{c}}\left[\frac{\beta}{\gamma_{g}}\left\langle\square_{s}\right\rangle\left(\frac{1}{\beta} \frac{\partial \beta}{\partial \xi^{+}}-\frac{1}{\gamma_{g}} \frac{\partial \gamma_{g}}{\partial \xi^{+}}\right)+\beta \gamma_{g}\left\langle\square_{t}\right\rangle\left(\frac{1}{\beta} \frac{\partial \beta}{\partial \xi^{+}}+\frac{1}{\gamma_{g}} \frac{\partial \gamma_{g}}{\partial \xi^{+}}\right)\right] \\
& -\frac{1}{N_{s}^{3} a_{s}^{3} N_{t} a_{t}}\left[\gamma_{q} \kappa\left(\frac{1}{\gamma_{q}} \frac{\partial \gamma_{q}}{\partial \xi^{+}}\right)\left\langle\bar{\psi} D_{0} \psi\right\rangle-\kappa\left(\frac{1}{\kappa} \frac{\partial \kappa}{\partial \xi^{+}}\right)\left(4 N_{c} N_{f}+\langle\bar{\psi} \psi\rangle\right)\right]+\mu_{q} n_{q} .
\end{aligned}
$$

The trace anomaly and pressure follow a similar procedure using the respective Karsch coefficients ( or $\beta$ functions) :

$$
\epsilon-3 p=\frac{T}{V}\left\langle a \frac{\partial S}{\partial a}\right\rangle, \quad p=-\frac{T}{3 V}\left[\left\langle a \frac{\partial S}{\partial a}\right\rangle+\left\langle\xi \frac{\partial S}{\partial \xi}\right\rangle\right]+\frac{\mu n_{q}}{3} .
$$

The angled brackets are vacuum subtracted using results from an ensemble with volume $16^{3} \times 24, j a=0.0$ and $\mu=0.0$. We calculate the energy density, quark number density, trace anomaly and pressure on 3 volumes $12^{3} \times 24,16^{3} \times 12$ and $16^{3} \times 8$, which translate to $47 \mathrm{MeV}$, $94 \mathrm{MeV}$ and $141 \mathrm{MeV}$ respectively. On all three volumes we measure the thermodynamic quantity in question at diquark source $j a=0.04$ and $j a=0.02$ and extrapolate to zero.

For the energy density, trace anomaly and the pressure we also repeated the analysis with 100 bootstrap sample values from the Karsch coefficient determination to estimate their uncertainty, shown as shaded symbols and dashed error bars. The quark number density (Fig 3, left) is shown to highlight the dominance of the quark number density term in both the energy density and the pressure. The energy density (Fig 3, right) is seen to be almost oblivious to the error coming from the Karsch coefficient determination except for small $\mu$. The quark number density shown in Figure 3 is normalised by $n_{S B}^{\text {cont }}$ and to allow for comparison with a previous calculation using the integral method (semi-filled symbols) [2], the pressure shown in Figure 4 is normalised by $p_{S B}^{\text {cont } t}$ :

$$
n_{S B}^{\text {cont }}=N_{f} N_{c}\left(\frac{\mu T^{2}}{3}+\frac{\mu^{3}}{3 \pi^{2}}\right), \quad p_{S B}^{\text {cont }}=\frac{N_{f} N_{c}}{12 \pi^{2}}\left(\mu^{4}+2 \pi^{2} \mu^{2} T^{2}+\frac{7 \pi^{4}}{15} T^{4}\right) .
$$



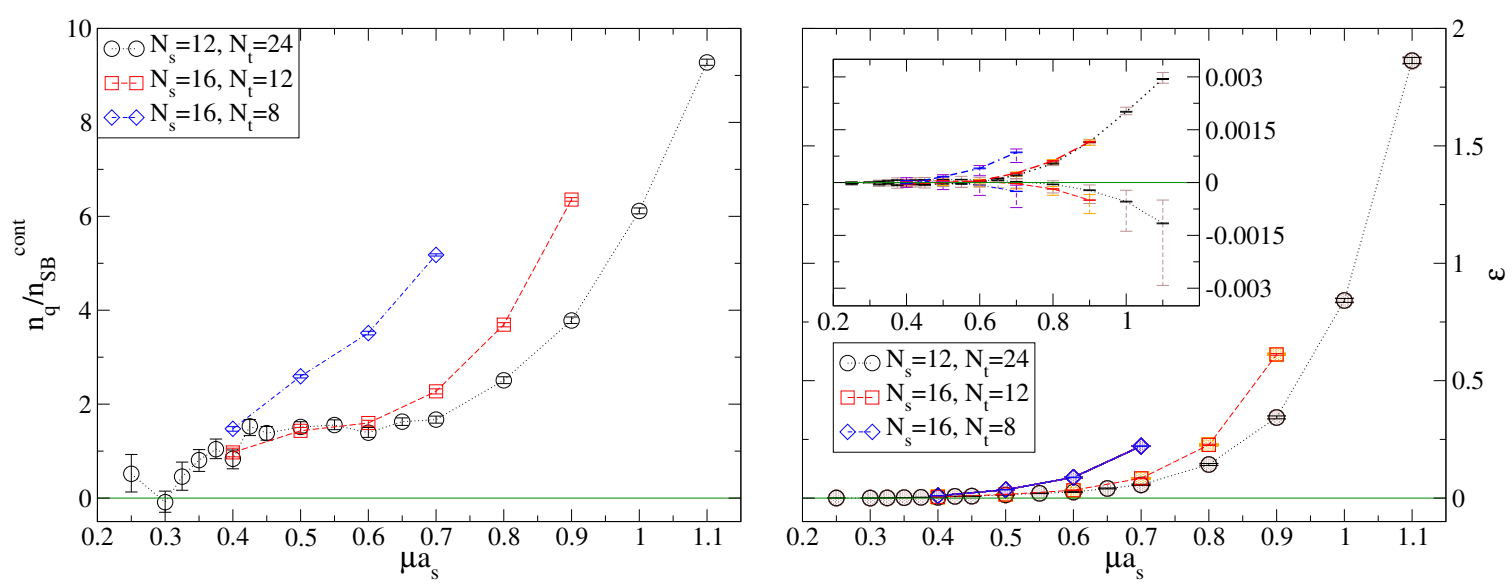

Figure 3: Quark number density (left), and total energy density (right) as a function of chemical potential $\mu$. Inset is the fermionic (bold) and gluonic contributions which come with a Karsch coefficient prefactor. The shaded symbols in the main plot and the dashed error bars in the inset denote the Karsch coefficient determination uncertainty.
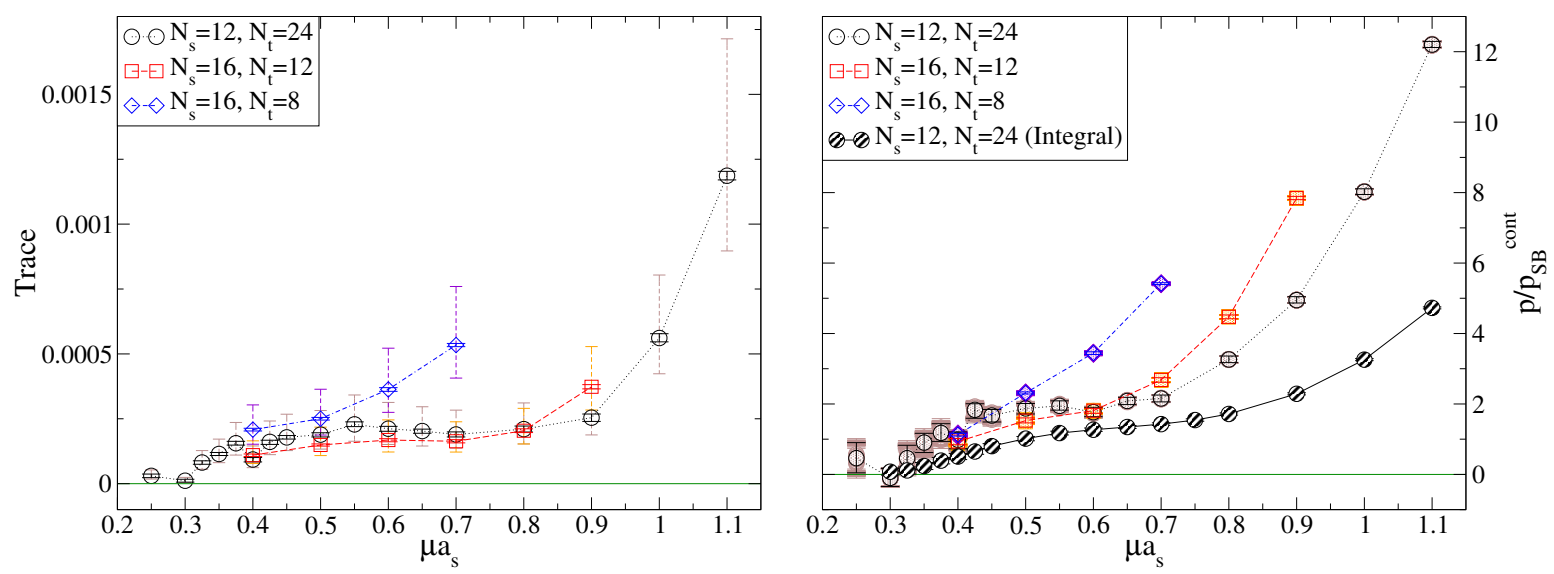

Figure 4: Trace Anomaly (left) and pressure (right) as a function of chemical potential $\mu$. The shaded symbols and dashed error bars denote the uncertainty coming from the Karsch coefficients determination. For the pressure, the results calculated using the integral method are also shown (semi-filled symbols).

The trace anomaly (Fig 4, left) remains positive, slowly rising at large $\mu$. As the trace anomaly is closer to zero in size, the uncertainty in the Karsch coefficients is more apparent. The pressure (Fig 4, right) is somewhat more sensitive to the difference in values of the Karsch coefficients at small $\mu$. The values generated on the $12^{3} \times 24$ volume, agree well with the results from the integral method. As the quark number density does not require the Karsch coefficients it can be seen that at higher chemical potential $\mu$, both the pressure and energy density are effectively Karsch coefficient independent. 


\section{Summary and Outlook}

This study demonstrates the feasibility of the derivative method using non-perturbatively determined Karsch coefficients. Several improvements are still possible. Our current static potential code doesn't scale well, and with new ensembles on larger volumes with finer lattice spacings coming online soon, a newer more efficient plan of attack is needed. At the moment that looks to be the $W_{0}$ scale from the Wilson flow [6] which we are currently working on changing to handle $S U(2)$ configurations rather than the $S U(3)$ ones it was designed for. This would also in principle replace the sideways potential code with which we used to calculate $\xi_{g}$, although possessing alternative methods and codes to measure the same quantity allows to control systematic uncertainties.

\section{Acknowledgments}

This project was part of the UKQCD collaboration and the DiRAC Facility jointly funded by STFC, the Large Facilities Capital Fund of BIS and Swansea University. We thank the DEISA Consortium, funded through the EU FP7 project RI222919, for support within the DEISA Extreme Computing Initiative. The simulation code was adapted with the help of Edinburgh Parallel Computing Centre funded by a grant from EPSRC. JIS and SC acknowledge the support of Science Foundation Ireland grants 08-RFP-PHY1462, 11RFP.1-PHY3193 and 11-RFP.1-PHY3193-STTF-1.

\section{References}

[1] Ludmila Levkova, Thomas Manke, and Robert Mawhinney. Two-flavor QCD thermodynamics using anisotropic lattices. Phys.Rev., D73:074504, 2006. [hep-lat/0603031].

[2] Seamus Cotter, Pietro Giudice, Simon Hands, and Jon-Ivar Skullerud. Towards the phase diagram of dense two-color matter. Phys.Rev., D87(3):034507, 2013. [arXiv:1210.4496].

[3] Simon Hands, Seyong Kim, and Jon-Ivar Skullerud. Deconfinement in dense 2-color QCD. Eur.Phys.J., C48:193, 2006. [hep-lat/0604004].

[4] Timothy R. Klassen. The Anisotropic Wilson gauge action. Nucl.Phys., B533:557-575, 1998. [hep-lat/9803010].

[5] Nikitas Stamatopoulos. Three Dimensional Lattice Dispersion Relations for Finite Difference Methods in Scalar Field Simulations. 2012. [arXiv:1210.3368].

[6] Szabolcs Borsányi, Stephan Dürr, Zoltán Fodor, Christian Hoelbling, Sándor D. Katz, et al. High-precision scale setting in lattice QCD. JHEP, 1209:010, 2012. [arXiv:1203.4469]. 